\title{
EXACT BOUNDARY CONTROLLABILITY OF COUPLED HYPERBOLIC EQUATIONS
}

\author{
SERGEI AVDONIN* ${ }^{*}$ AbDon CHOQUE RIVERO **, LUZ DE TERESA *** \\ * Department of Mathematics and Statistics \\ University of Alaska Fairbanks, Fairbanks, AK 99775-6660, USA \\ e-mail: S.avdonin@alaska.edu \\ ${ }^{* *}$ Institute of Physics and Mathematics \\ Universidad Michoacana de San Nicolás de Hidalgo Ciudad Universitaria, Ed. C-3, C.P. 58040 Morelia, Mich., México \\ e-mail: abdoneifm.umich.mx \\ *** Institute of Mathematics \\ Universidad Nacional Autonoma de México, Circuito Exterior, C.U. 04510 D.F., México \\ e-mail: deteresa@matem.unam.mx
}

\begin{abstract}
We study the exact boundary controllability of two coupled one dimensional wave equations with a control acting only in one equation. The problem is transformed into a moment problem. This framework has been used in control theory of distributed parameter systems since the classical works of A.G. Butkovsky, H.O. Fattorini and D.L. Russell in the late 1960s to the early 1970s. We use recent results on the Riesz basis property of exponential divided differences.
\end{abstract}

Keywords: coupled wave equations, controllability, Riesz bases, moment problem.

\section{Introduction}

In the last fifteen years an increasing interest has been observed in the study of the controllability of coupled equations of the same nature, exerting control on fewer equations that the total number of equations in the system. Great effort has been observed in the study of coupled parabolic equations (see, e.g., the work of Ammar-Kohdja et al. (2011) and the references therein) but very little has been said about the controllability of coupled wave equations.

As far as we know, only five papers have been published treating this interesting problem (Dáger, 2006; Tebou, 2008; Alabau-Boussouira, 2003), and two are very recent results (Alabau-Boussouira and Leautaud, 2011; Rosier and de Teresa, 2011). The first two papers deal with the so-called "insensitizing controls", a concept introduced by Lions (1989) that can be transformed in the controllability of a cascade system of two equations with the particularity that the only controlled state is "the second" and this second equation is a backward equation with zero initial data (Lions, 1989; Bodart and Fabre, 1995; de Teresa, 2000; Dáger, 2006; Tebou, 2008).
More precisely, in the case of the wave equation, the following problem is treated. For $\Omega$ an open and bounded set of $\mathbb{R}^{N}, \omega, \mathcal{O} \subset \Omega$ non-empty subsets and $T>0$, we consider the coupled system

$$
\begin{aligned}
& \begin{cases}y_{t t}-\Delta y=h \chi_{\omega} & \text { in } Q:=\Omega \times(0, T), \\
y=0 & \text { on } \Sigma:=\partial \Omega \times(0, T), \\
y(x, 0)=y^{0}(x), & \text { in } \Omega \\
y_{t}(x, 0)=y^{1}(x) & \text { in } \Omega,\end{cases} \\
& \begin{cases}q_{t t}-\Delta q=y \chi_{\mathcal{O}} & \text { in } Q, \\
q=0 & \text { on } \Sigma, \\
q(x, T)=0, \quad q_{t}(x, T)=0 & \text { in } \Omega .\end{cases}
\end{aligned}
$$

The problem is now to give conditions on $\omega, \mathcal{O}$ and $T>0$ to guarantee that for every $\left(y^{0}, y^{1}\right) \in L^{2}(\Omega) \times H^{-1}(\Omega)$ there exists a control $h$ in an appropriate space, such that

$$
q(x, 0)=q_{t}(x, 0)=0 .
$$

This problem was solved in the one dimensional case by Dáger (2006) and in the $n$-dimensional case by Tebou (2008). A controllability result for a system similar to (1), (2) in the one dimensional case is also presented by Rosier and de Teresa (2011). 
On the other hand, using a two level energy method, Alabau-Boussouira (2003) solved, for a small parameter $\alpha>0$, the following boundary controllability problem. Given any initial $\left(y^{0}, q^{0}, y^{1}, q^{1}\right)$ and final data $\left(y^{0, T}, q^{0, T}, y^{1, T}, q^{1, T}\right)$ both in

$$
\left.\mathcal{V}:=L^{2}(\Omega) \times H_{0}^{1}(\Omega) \times H^{-1}(\Omega) \times L^{2}(\Omega)\right),
$$

the author described conditions on $\Gamma_{0}$ and $T>0$ that guarantee the existence of $v \in L^{2}(\Sigma)$ such that the corresponding solution to

$$
\begin{cases}y_{t t}-\Delta y+\alpha q=0 & \text { in } Q, \\ q_{t t}-\Delta q+\alpha y=0 & \text { in } Q, \\ y=v & \text { on } \Gamma_{0} \times(0, T), \\ y=0 & \text { on } \Gamma_{1} \times(0, T), \\ q=0 & \text { on } \Sigma, \\ y(x, 0)=y^{0}(x) & \text { in } \Omega, \\ y_{t}(x, 0)=y^{1}(x) & \text { in } \Omega, \\ q(x, 0)=q^{0}(x), & \text { in } \Omega, \\ q_{t}(x, 0)=q^{1}(x) & \text { in } \Omega\end{cases}
$$

satisfies

$$
\left(y(T), q(T), y_{t}(T), q_{t}(T)=\left(y^{0, T}, q^{0, T}, y^{1, T}, q^{1, T}\right) .\right.
$$

In the work of Alabau-Boussouira and Leautaud (2011) a generalization of this result is given, but again, the result is proved for a small coupling parameter.

We notice that controllability and observability questions for coupled partial differential equations are closely related to source identification and sensor location problems for distributed parameter systems (see, e.g., Khapalov, 2010; Uciński and Patan, 2010; El Jai and Hamzaoui, 2009). These models also arise in a very important applied area-wave propagation in porous media (Boit, 1962; Holger et al., 2010). Stabilizability of coupled wave equations and its applications are discussed by Najafi et al. (1997) and Najafi (2001).

In this paper we work in the one-dimensional model. We improve results existing in the literature for the case of one boundary control of two coupled hyperbolic equations. We demonstrate that the condition imposed by Alabau-Boussouira (2003) as well as Alabau-Boussouira and Leautaud (2011) on the "smallness" of the coupling parameter can be eliminated. Instead of the smallness, we obtain explicit analytic conditions on the coupling parameters necessary and sufficient for the exact controllability of the system. These conditions have a spectral character, and for a given system, there is only a finite number of parameters that violate the conditions.

Let $\Omega=(0, \pi), T>0, Q=\Omega \times(0, T)$ and $\alpha, \beta \in$
$\mathbb{R}$. We study controllability properties of the system

$$
\begin{cases}y_{t t}-y_{x x}+\alpha q=0 & \text { in } Q, \\ q_{t t}-q_{x x}+\beta y=0 & \text { in } Q, \\ y(0, t)=u(t), y(\pi, t)=0 & t \in(0, T) \\ q=0 & \text { on } \Sigma, \\ y(x, 0)=y^{0}(x) & \text { in } \Omega, \\ y_{t}(x, 0)=y^{1}(x) & \text { in } \Omega, \\ q(x, 0)=q^{0}(x), & \text { in } \Omega, \\ q_{t}(x, 0)=q^{1}(x) & \text { in } \Omega .\end{cases}
$$

We will also consider the particular case in which $\alpha=0$ for $Q=(0, \pi) \times(0, T)$,

$$
\begin{cases}y_{t t}-y_{x x}=0, \text { in } Q & \\ q_{t t}-q_{x x}+\beta y=0 & \text { in } Q, \\ y(0, t)=u(t), y(\pi, t)=0 & t \in(0, T) \\ q=0 & \text { on } \Sigma, \\ y(x, 0)=y^{0} & \text { in }(0, \pi), \\ y_{t}(x, 0)=y^{1}(x) & \text { in }(0, \pi), \\ q(x, 0)=q^{0}(x) & \text { in } \Omega, \\ q_{t}(x, 0)=q^{1}(x) & \text { in } \Omega .\end{cases}
$$

This last case is especially important to us because, on the one hand, it is related to insensitizing results (and gives a different proof of the results of Rosier and de Teresa (2011)) and, on the other hand, it reveals the main ideas behind the rest of the proofs in this paper. In fact, we first prove the controllability result for (5), where we use the properties of exponential divided differences of a special form, $\left\{\exp \left(i \omega_{n} t\right), t \exp \left(i \omega_{n} t\right)\right\}$. The key point to solve the controllability problem for (4) is to use the results of Avdonin and Ivanov (2001) as well as Avdonin and Moran (2001a), who presented a study of exponential divided differences of a general form.

To prove our results, we apply the method of moments. It has been widely used in the control theory of distributed parameter systems since the classical papers of H.O. Fattorini and D.L. Russell in the late 1960s to early 1970s (see the excellent survey by Russell (1978) and the book by Avdonin and Ivanov (1995) for the history of the subject and complete references). The method is based on properties of exponential families (usually in the space $\left.L^{2}(0, T)\right)$, the most important of which for control theory are minimality, the Riesz basis property and also the $\mathcal{L}$-basis property. The latter is defined to be a Riesz basis in the closure of the linear span of the family. Recent investigations into new classes of distributed systems such as hybrid systems, systems with memory, and damped systems as well as problems of simultaneous control have raised a number of new and difficult problems in the theory of exponential families (see, e.g., Hansen and Zuazua, 1995; Avdonin and Moran, 2001b; Pandolfi, 2009; Avdonin and Pandolfi, 2011) including the theory of vector-valued exponentials (Avdonin and Ivanov, 1995) and many other interesting topics. 
As mentioned above, we study the controllability properties of the system (4). We suppose that $u \in$ $L^{2}(0, T)$ and then set $\mathcal{H}_{0}=L^{2}(0, \pi), \quad \mathcal{H}_{1}=$ $H_{0}^{1}(0, \pi), \mathcal{H}_{-1}=H^{-1}(0, \pi)$ and $\mathcal{V}=\mathcal{H}_{0} \times \mathcal{H}_{1} \times$ $\mathcal{H}_{-1} \times \mathcal{H}_{0}$. Our important auxiliary result establishes the regularity of the solution to the IBVP (4).

Theorem 1. Let $\left(y^{0}, q^{0}, y^{1}, q^{1}\right) \in \mathcal{V}$. Then the solution pair $(y, q)=\left(y^{u}(x, t), q^{u}(x, t)\right)$ of the IBVP (4) exists, is unique and satisfies the inclusion $\left(y, q, y_{t}, q_{t}\right) \in C([0, T] ; \mathcal{V})$. This means that for any $t \in[0, T],\left(y(\cdot, t), q(\cdot, t), y_{t}(\cdot, t), q_{t}(\cdot, t)\right) \in \mathcal{V}$ and is continuous in $t$ in the norm on $\mathcal{V}$.

\section{Definition 1.}

(i) The system (4) is called exactly controllable in the time interval $[0, T]$ if, for any $\left(y^{0}, q^{0}, y^{1}, q^{1}\right) \in \mathcal{V}$, the reachable set

$$
\left\{\left(y^{u}(\cdot, T), q^{u}(\cdot, T), y_{t}^{u}(\cdot, T), q_{t}^{u}(\cdot, T)\right): u \in L^{2}(0, T)\right\}
$$

is equal to $\mathcal{V}$.

(ii) The system (4) is called approximately controllable in the time interval $[0, T]$ if, for any $\left(y^{0}, y^{1}, q^{0}, q^{1}\right) \in \mathcal{V}$, the reachable set

$$
\left\{\left(y^{u}(\cdot, T), q^{u}(\cdot, T), y_{t}^{u}(\cdot, T), q_{t}^{u}(\cdot, T)\right): u \in L^{2}(0, T)\right\}
$$

is dense in $\mathcal{V}$.

The main results of this paper are described by the following theorem.

Theorem 2. Suppose $\beta \neq 0$, and consider the equality

$$
n^{2}-\sqrt{\alpha \beta}=m^{2}+\sqrt{\alpha \beta}, \quad m, n \in \mathbb{N} .
$$

(i) If (6) never occurs for $m \neq n$, the system (4) is exactly controllable in the time interval $[0, T], T \geq 4 \pi$.

(ii) If (6) occurs for some $m, n$, the system (4) is not approximately controllable for any $T$.

(iii) The system (4) is not approximately controllable for $T<4 \pi$.

Remark 1. If $\beta=0$, then the second equation is decoupled from the first equation, where the control acts and therefore system (4) is not approximately controllable for any $T>0$.

Remark 2. Observe that equality (6) holds if $\alpha \beta=p^{2} / 4$ for some $p \in \mathbb{N}$. In particular, it never holds if $|\alpha \beta|$ is small enough, more precisely, if $|\alpha \beta|<1 / 4$. Therefore, our results agree with those of Alabau-Boussouira (2003) as well as Alabau-Boussouira and Leautaud (2011) for the one dimensional setting.

We notice that the system (4) is symmetric with respect to $y$ and $q$, but control $u$ appears in an asymmetric way. We formulate separately the important particular case (for $\alpha=0$ ) of Theorem 2
Theorem 3. If $\beta \neq 0$, the system (5) is exactly controllable for $T \geq 4 \pi$.

The technique used in the proof of this theorem allows us to strengthen the last result:

Theorem 4. Let $\beta \neq 0$ and $\mathcal{O}=(a, b) \subset(0, \pi)$. If we replace $\beta$ by $\beta \chi_{\mathcal{O}}$ in the second equation of (5), the system (5) is exactly controllable for $T \geq 4 \pi$.

The rest of the paper is organized as follows. In Section 2 we reduce the controllability problem to a moment problem. In Section 3 we solve this moment problem. In Section 3.1 we consider the case $\alpha=0$, in Sections 3.2 and 3.3 we consider the cases $\alpha \beta>0$ and $\alpha \beta<0$, correspondingly. In Section 4 we prove Theorems 13 .

\section{From the coupled system to the moment problem}

To prove Theorem 2] we transform the controllability problem into a moment problem of the form

$$
\int_{0}^{T} u(t) f_{n}(t) \mathrm{d} t=c_{n}, \quad n \in \mathbb{N}
$$

It is known (see Avdonin and Ivanov, 1995, Ch. I.2) that the problem (7) has a solution $u \in L^{2}(0, T)$ for any $\left\{c_{n}\right\}_{n \in \mathbb{N}} \in \ell^{2}(\mathbb{R})$ if and only if the family $\mathcal{F}=\left\{f_{n}\right\}_{n \in \mathbb{N}}$ forms an $\mathcal{L}$-basis in $L^{2}(0, T)$. The family $\mathcal{F}$ has different forms in the cases $\alpha=0, \alpha \beta>0$ and $\alpha \beta<0$. We prove that in all these cases, when (6) does not hold (it may hold if $\alpha \beta>0$ ), the corresponding family $\mathcal{F}$ forms a Riesz basis in $L^{2}(0, T)$ for $T \geq 4 \pi$.

We start with deriving the problem of moments related to the exact controllability of the system (4). It is well known that the reversibility and linearity of the system (4) makes the exact controllability equivalent to the exact controllability from zero. Therefore, we consider the system

$$
\left\{\begin{array}{l}
y_{t t}-y_{x x}+\alpha q=0, \quad 0<x<\pi, \quad 0<t<T \\
q_{t t}-q_{x x}+\beta y=0, \\
y(0, t)=u(t), \quad y(\pi, t)=q(0, t)=q(\pi, t)=0 \\
y(x, 0)=y_{t}(x, 0)=q(x, 0)=q_{t}(x, 0)=0
\end{array}\right.
$$

Here $\alpha, \beta \in \mathbb{R}$, with $\beta \neq 0$ and $u \in L^{2}(0, T)$.

We present $y, q$ in the form of series

$$
y(x, t)=\sum_{n=1}^{\infty} a_{n}(t) \phi_{n}(x), \quad q(x, t)=\sum_{n=1}^{\infty} b_{n}(t) \phi_{n}(x),
$$

where $\phi_{n}(x)=\sqrt{2 / \pi} \sin (n x)$. Multiplying (8) by 
$\sin n x$ and integrating by parts in $(0, \pi)$, we obtain

$$
\left\{\begin{array}{l}
\int_{0}^{\pi}\left(y_{t t}(x, t)+n^{2} y(x, t)+\alpha q(x, t)\right) \sin n x \mathrm{~d} x \\
=n u(t), \quad 0<t<T \\
\int_{0}^{\pi}\left(q_{t t}(x, t)+n^{2} q(x, t)+\beta y(x, t)\right) \sin n x \mathrm{~d} x \\
=0, \quad 0<t<T .
\end{array}\right.
$$

Substituting (9) in (10), we obtain

$$
\left\{\begin{array}{l}
\ddot{a}_{n}+n^{2} a_{n}+\alpha b_{n}=\kappa_{n} u(t), \quad \kappa_{n}=\sqrt{\frac{2}{\pi}} n \\
\ddot{b}_{n}+n^{2} b_{n}+\beta a_{n}=0 \\
a_{n}(0)=\dot{a}_{n}(0)=b_{n}(0)=\dot{b}_{n}(0)=0
\end{array}\right.
$$

To solve the system (11) for every $n$, we set

$$
Y_{n}=\left(\begin{array}{c}
a_{n} \\
b_{n} \\
\dot{a}_{n} \\
\dot{b}_{n}
\end{array}\right), \quad A_{n}=\left(\begin{array}{cccc}
0 & 0 & 1 & 0 \\
0 & 0 & 0 & 1 \\
-n^{2} & -\alpha & 0 & 0 \\
-\beta & -n^{2} & 0 & 0
\end{array}\right)
$$

and rewrite 11 in the form

$$
\dot{Y}_{n}(t)=A_{n} Y_{n}(t)+F_{n}(t), \quad Y_{n}(0)=0 .
$$

The solution to (12) can be written in the form

$$
\begin{aligned}
Y_{n}(t) & =\int_{0}^{t} e^{A_{n}(t-\tau)} F_{n}(\tau) \mathrm{d} \tau \\
F_{n}(t) & =\left(\begin{array}{c}
0 \\
0 \\
\kappa_{n} \\
0
\end{array}\right) u(t) .
\end{aligned}
$$

We use the finite power series representation of the matrix function $e^{A_{n} t}$ which crucially employs the Cayley-Hamilton theorem (see Leonard, 1996),

$$
\begin{aligned}
e^{A_{n} t}= & z_{0, n}(t) I+z_{1, n}(t) A_{n} \\
& +z_{2, n}(t) A_{n}^{2}+z_{3, n}(t) A_{n}^{3}
\end{aligned}
$$

where $z_{k, n}(t)$ are the solutions of the differential equations

$$
\begin{aligned}
z^{(4)}+2 n^{2} z^{\prime \prime}+n^{4}-\alpha \beta=0, \\
z_{k, n}^{(j)}(0)=\delta_{j k}, \quad j, k=0,1,2,3 .
\end{aligned}
$$

The characteristic polynomial associated to 15 is

$$
\begin{aligned}
& \lambda^{4}+2 n^{2} \lambda^{2}+n^{4}-\alpha \beta \\
& =\left(\lambda^{2}+n^{2}-\sqrt{\alpha \beta}\right)\left(\lambda^{2}+n^{2}+\sqrt{\alpha \beta}\right) .
\end{aligned}
$$

The roots of this polynomial determine the solutions $z_{k, n}(t)$. The controllability problem reduces then to the moment problem of the form

$$
\int_{0}^{T} e^{A_{n}(T-t)} F_{n}(t) \mathrm{d} t=c_{n}, \quad\left\{c_{n}\right\}_{n \in \mathbb{N}} \in \ell^{2}\left(\mathbb{R}^{4}\right) .
$$

In the following sections we present the explicit form of this moment problem and derive its solution depending on the parameters $\alpha$ and $\beta$.

\section{Solution of the moment problem}

It is well known (see, e.g., Avdonin and Ivanov, 1995, Sec. III.1) that the norms of the functions $y$ and $q$ as well as their time derivatives in the corresponding Sobolev spaces are equivalent to the norms of their Fourier coefficients in the weighted $\ell^{2}$ spaces:

$$
\begin{aligned}
& \left\|\left(y^{u}(\cdot, T), q^{u}(\cdot, T), y_{t}^{u}(\cdot, T), q_{t}^{u}(\cdot, T)\right)\right\|_{\mathcal{V}}^{2} \\
& \quad \asymp \sum_{n=1}^{\infty}\left[\left|a_{n}(T)\right|^{2}+\left|n b_{n}(T)\right|^{2}+\left|n^{-1} \dot{a}_{n}(T)\right|^{2}\right. \\
& \left.\quad+\left|\dot{b}_{n}(T)\right|^{2}\right] .
\end{aligned}
$$

Solutions of Eqns. (11) and (12) depend on $\alpha$ and $\beta$. We discuss three different cases.

3.1. Case $\boldsymbol{\alpha}=\mathbf{0}, \boldsymbol{\beta} \neq \mathbf{0}$. It can be easily checked that the solution of (11) and (12), in the case $\alpha=0$, is given by the formulas

$$
\begin{aligned}
a_{n}(t)= & \frac{\kappa_{n}}{n} \int_{0}^{t} \sin (n(t-\tau)) u(\tau) \mathrm{d} \tau \\
b_{n}(t)= & -\frac{\kappa_{n} \beta}{2 n^{3}} \int_{0}^{t}(\sin (n(t-\tau)) \\
& -n(t-\tau) \cos (n(t-\tau))) u(\tau) \mathrm{d} \tau, \\
\dot{a}_{n}(t)= & \kappa_{n} \int_{0}^{t} \cos (n(t-\tau)) u(\tau) \mathrm{d} \tau \\
\dot{b}_{n}(t)= & -\frac{\kappa_{n} \beta}{2 n} \int_{0}^{t}(t-\tau) \sin (n(t-\tau)) u(\tau) \mathrm{d} \tau .
\end{aligned}
$$

Since $\kappa_{n}=\sqrt{2 / \pi} n$, we can write

$$
\begin{gathered}
a_{n}(t)=\sqrt{\frac{2}{\pi}} \int_{0}^{t} \sin (n(t-\tau)) u(\tau) \mathrm{d} \tau, \\
n b_{n}(t)=-\frac{\beta}{\sqrt{2 \pi}} \int_{0}^{t}\left(\frac{\sin (n(t-\tau))}{n}\right. \\
-(t-\tau) \cos (n(t-\tau))) u(\tau) \mathrm{d} \tau, \\
n^{-1} \dot{a}_{n}(t)=\sqrt{\frac{2}{\pi}} \int_{0}^{t} \cos (n(t-\tau)) u(\tau) \mathrm{d} \tau, \\
\dot{b}_{n}(t)=-\frac{\beta}{\sqrt{2 \pi}} \int_{0}^{t}(t-\tau) \\
\quad \times \sin (n(t-\tau)) u(\tau) \mathrm{d} \tau .
\end{gathered}
$$


Our next step is to prove that the family of functions

$$
\begin{gathered}
\mathcal{F}=\left\{\sqrt{\frac{2}{\pi}} \sin n t, \frac{\beta}{\sqrt{2 \pi}}\left(-\frac{\sin n t}{n}+t \cos n t\right),\right. \\
\left.\sqrt{\frac{2}{\pi}} \cos n t,-\frac{\beta}{\sqrt{2 \pi}} t \sin n t\right\}, \quad n \in \mathbb{N},
\end{gathered}
$$

forms an $\mathcal{L}$-basis in $L^{2}(0, T)$ for $T \geq 4 \pi$. Recall that a family is said to be an $\mathcal{L}$-basis if it forms a Riesz basis in the closure of its linear span.

It is known (see, e.g., Avdonin and Ivanov, 1995; 2001; Avdonin and Moran, 2001a, Section II.4.5) that the family

$$
\mathcal{E}=\{\sin n t, t \cos n t, \cos n t, t \sin n t,\}, \quad n \in \mathbb{N},
$$

forms an $\mathcal{L}$-basis in $L^{2}(0, T)$ for $T \geq 4 \pi$. It is easy to check that the operator $\mathcal{B}$ which maps $\mathcal{E}$ to $\mathcal{F}$ is bounded and boundedly invertible in $L^{2}(0, T)$ for any $T>0$ since its matrix form can be presented as

$$
\mathcal{B}=\operatorname{diag}\left(B_{n}\right)_{n=1}^{\infty},
$$

with

$$
\begin{gathered}
B_{n}=\left(\begin{array}{cccc}
\sqrt{\frac{2}{\pi}} & 0 & 0 & 0 \\
-\frac{\beta}{n \sqrt{2 \pi}} & \frac{\beta}{\sqrt{2 \pi}} & 0 & 0 \\
0 & 0 & \sqrt{\frac{2}{\pi}} & 0 \\
0 & 0 & 0 & -\frac{\beta}{\sqrt{2 \pi}}
\end{array}\right), \\
B_{n}^{-1}=\left(\begin{array}{cccc}
\sqrt{\frac{\pi}{2}} & 0 & 0 & 0 \\
\frac{1}{n} \sqrt{\frac{\pi}{2}} & \frac{\sqrt{2 \pi}}{\beta} & 0 & 0 \\
0 & 0 & \sqrt{\frac{\pi}{2}} & 0 \\
0 & 0 & 0 & -\frac{\sqrt{2 \pi}}{\beta}
\end{array}\right) .
\end{gathered}
$$

Therefore, $\mathcal{F}$ is also an $\mathcal{L}$-basis in $L^{2}(0, T)$ for $T \geq 4 \pi$.

Remark 3. The presented proof can be extended to the case when instead of $\beta$ we take $\beta \chi_{\mathcal{O}}$ as in Theorem 4. Only the expression of the moment problem becomes more complicated but the method of its solution is exactly the same. We skip the routine details of the proof and refer to the works of Rosier and de Teresa (2011) as well as Kavian and de Teresa (2010) for related results.

3.2. Case $\boldsymbol{\alpha} \boldsymbol{\beta}>\mathbf{0}$. In this section we consider the case in which both the coupling parameters have the same sign. As previously, we want to solve a problem of moments. To this end, we need to construct the corresponding Riesz basis of exponentials. In general (see below), this basis is given by two families of functions. The first one is defined by a finite number of indexes $n$ : $n^{2}<\sqrt{\alpha \beta}$, and the second-by an infinite number of indexes $n: n^{2} \geq \sqrt{\alpha \beta}$. Clearly, when $\alpha \beta \leq 1$, we only have the second family indexed by $n \in \mathbb{N}$. We start with the study of the finite family.

Subcase $n^{2}<\sqrt{\boldsymbol{\alpha} \beta}$. There can be a finite number of $n$ satisfying this inequality. In this case, the characteristic polynomial of matrix $A_{n}$ is given by

$$
\begin{aligned}
\lambda^{4}+2 n^{2} \lambda^{2}+n^{4}- & \alpha \beta \\
& =\left(\lambda^{2}+\left(\gamma_{n}^{+}\right)^{2}\right)\left(\lambda^{2}-\left(\gamma_{n}^{-}\right)^{2}\right)
\end{aligned}
$$

where

$$
\gamma_{n}^{+}=\sqrt{n^{2}+\sqrt{\alpha \beta}}, \quad \gamma_{n}^{-}=\sqrt{\sqrt{\alpha \beta}-n^{2}} .
$$

In this case the solution of (11) and (12) has the form

$$
\begin{aligned}
& a_{n}(t) \\
& =\frac{\kappa_{n}}{2} \int_{0}^{t}\left(\frac{\sinh \left[(t-\tau) \gamma_{n}^{-}\right]}{\gamma_{n}^{-}}+\frac{\sin \left[(t-\tau) \gamma_{n}^{+}\right]}{\gamma_{n}^{+}}\right) \\
& \quad \times u(\tau) \mathrm{d} \tau, \\
& b_{n}(t) \\
& =\frac{\kappa_{n}}{2} \sqrt{\frac{\beta}{\alpha}} \int_{0}^{t}\left(\frac{\sin \left[(t-\tau) \gamma_{n}^{+}\right]}{\gamma_{n}^{+}}-\frac{\sinh \left[(t-\tau) \gamma_{n}^{-}\right]}{\gamma_{n}^{-}}\right) \\
& \quad \times u(\tau) \mathrm{d} \tau, \\
& \dot{a}_{n}(t) \\
& =\frac{\kappa_{n}}{2} \int_{0}^{t}\left(\cos \left[(t-\tau) \gamma_{n}^{+}\right]+\cosh \left[(t-\tau) \gamma_{n}^{-}\right]\right) u(\tau) \mathrm{d} \tau, \\
& \dot{b}_{n}(t) \\
& =\frac{\kappa_{n}}{2} \sqrt{\frac{\beta}{\alpha}} \int_{0}^{t}\left(\cos \left[(t-\tau) \gamma_{n}^{+}\right]-\cosh \left[(t-\tau) \gamma_{n}^{-}\right]\right) \\
& \quad \times u(\tau) \mathrm{d} \tau .
\end{aligned}
$$

We consider the finite family

$$
\begin{aligned}
\mathcal{F}_{\sqrt{\alpha \beta}} & \left\{\frac{n}{\sqrt{2 \pi}}\left(\frac{\sinh \left[(t-\tau) \gamma_{n}^{-}\right]}{\gamma_{n}^{-}}+\frac{\sin \left[(t-\tau) \gamma_{n}^{+}\right]}{\gamma_{n}^{+}}\right),\right. \\
& \frac{n^{2}}{\sqrt{2 \pi}}\left(\frac{\sin \left[(t-\tau) \gamma_{n}^{+}\right]}{\gamma_{n}^{+}}-\frac{\sinh \left[(t-\tau) \gamma_{n}^{-}\right]}{\gamma_{n}^{-}}\right), \\
& \frac{1}{\sqrt{2 \pi}}\left(\cos \left[(t-\tau) \gamma_{n}^{+}\right]+\cosh \left[(t-\tau) \gamma_{n}^{-}\right]\right), \\
& \left.\frac{n}{\sqrt{2 \pi}}\left(\cos \left[(t-\tau) \gamma_{n}^{+}\right]-\cosh \left[(t-\tau) \gamma_{n}^{-}\right]\right)\right\}_{n^{2} \leq \sqrt{\alpha \beta}}
\end{aligned} .
$$
family

It is clear that the map that transforms $\mathcal{F}_{\sqrt{\alpha \beta}}$ into the

$$
\mathcal{E}_{\sqrt{\alpha \beta}}=\left\{e^{-i\left(i \gamma_{n}^{-} t\right)}, e^{i\left(i \gamma_{n}^{-} t\right)}, e^{i \gamma_{n}^{+} t}, e^{-i \gamma_{n}^{+} t}\right\}_{n^{2} \leq \sqrt{\alpha \beta}}
$$

is bounded and boundedly invertible in $L^{2}(0, T)$.

Subcase $\boldsymbol{n}^{\mathbf{2}} \geq \sqrt{\boldsymbol{\alpha} \boldsymbol{\beta}}$. We define $\omega_{n}^{ \pm}=\sqrt{n^{2} \pm \sqrt{\alpha \beta}}$. 
The solution of (11) and (12) is given by

$$
\begin{aligned}
& a_{n}(t) \\
& =\frac{\kappa_{n}}{2} \int_{0}^{t}\left(\frac{\sin \left(\omega_{n}^{-}(t-\tau)\right)}{\omega_{n}^{-}}+\frac{\sin \left(\omega_{n}^{+}(t-\tau)\right)}{\omega_{n}^{+}}\right) \\
& \quad \times u(\tau) \mathrm{d} \tau, \\
& b_{n}(t) \\
& =\frac{\kappa_{n}}{2} \sqrt{\frac{\beta}{\alpha}} \int_{0}^{t}\left(\frac{\sin \left(\omega_{n}^{+}(t-\tau)\right)}{\omega_{n}^{+}}-\frac{\sin \left(\omega_{n}^{-}(t-\tau)\right)}{\omega_{n}^{-}}\right) \\
& \quad \times u(\tau) \mathrm{d} \tau,
\end{aligned}
$$

$$
\begin{aligned}
& \dot{a}_{n}(t) \\
& =\frac{\kappa_{n}}{2} \int_{0}^{t}\left(\cos \left(\omega_{n}^{-}(t-\tau)\right)+\cos \left(\omega_{n}^{+}(t-\tau)\right)\right) u(\tau) \mathrm{d} \tau,
\end{aligned}
$$

$$
\begin{aligned}
& \dot{b}_{n}(t) \\
& =\frac{\kappa_{n}}{2} \sqrt{\frac{\beta}{\alpha}} \int_{0}^{t}\left(\cos \left(\omega_{n}^{+}(t-\tau)\right)-\cos \left(\omega_{n}^{-}(t-\tau)\right)\right) \\
& \quad \times u(\tau) \mathrm{d} \tau .
\end{aligned}
$$

If some $\omega_{n}^{-}=0$, we set

$$
\frac{\sin \omega_{n}^{-}(t-\tau)}{\omega_{n}^{-}}=t-\tau
$$

Remark 4. One can check that, as $\alpha \rightarrow 0$, we obtain in the limit the formulas of Section 3.1.

Substituting $\kappa_{n}=\sqrt{2 / \pi} n$, we have

$$
\begin{aligned}
a_{n}(t)= & \frac{n}{\sqrt{2 \pi}} \int_{0}^{t}\left(\frac{\sin \left(\omega_{n}^{-}(t-\tau)\right)}{\omega_{n}^{-}}\right. \\
& \left.+\frac{\sin \left(\omega_{n}^{+}(t-\tau)\right)}{\omega_{n}^{+}}\right) u(\tau) \mathrm{d} \tau, \\
n b_{n}(t)= & \frac{n^{2}}{\sqrt{2 \pi}} \sqrt{\frac{\beta}{\alpha}} \int_{0}^{t}\left(\frac{\sin \left(\omega_{n}^{+}(t-\tau)\right)}{\omega_{n}^{+}}\right. \\
& \left.-\frac{\sin \left(\omega_{n}^{-}(t-\tau)\right)}{\omega_{n}^{-}}\right) u(\tau) \mathrm{d} \tau, \\
n^{-1} \dot{a}_{n}(t)= & \frac{1}{\sqrt{2 \pi}} \int_{0}^{t}\left(\cos \left(\omega_{n}^{-}(t-\tau)\right)\right. \\
& \left.+\cos \left(\omega_{n}^{+}(t-\tau)\right)\right) u(\tau) \mathrm{d} \tau, \\
\dot{b}_{n}(t)= & \frac{n}{\sqrt{2 \pi}} \sqrt{\frac{\beta}{\alpha}} \int_{0}^{t}\left(\cos \left(\omega_{n}^{+}(t-\tau)\right)\right. \\
& \left.-\cos \left(\omega_{n}^{-}(t-\tau)\right)\right) u(\tau) \mathrm{d} \tau .
\end{aligned}
$$

Let us consider the family

$$
\begin{aligned}
\mathcal{E}_{1}= & \left\{\sin \left(\omega_{n}^{+} t\right), \frac{\sin \left(\omega_{n}^{+} t\right)-\sin \left(\omega_{n}^{-} t\right)}{\omega_{n}^{+}-\omega_{n}^{-}},\right. \\
& \left.\cos \left(\omega_{n}^{+} t\right), \frac{\cos \left(\omega_{n}^{+} t\right)-\cos \left(\omega_{n}^{-} t\right)}{\omega_{n}^{+}-\omega_{n}^{-}}\right\}_{n^{2} \geq \sqrt{\alpha \beta}} .
\end{aligned}
$$

One can check that the operator which maps $\mathcal{E}_{1}$ to the family

$$
\begin{aligned}
\mathcal{F}_{1}= & \left\{\frac{n}{\sqrt{2 \pi}}\left(\frac{\sin \left(t \omega_{n}^{-}\right)}{\omega_{n}^{-}}+\frac{\sin \left(t \omega_{n}^{+}\right)}{\omega_{n}^{+}}\right),\right. \\
& \frac{n^{2}}{\sqrt{2 \pi}} \sqrt{\frac{\beta}{\alpha}}\left(\frac{\sin \left(t \omega_{n}^{+}\right)}{\omega_{n}^{+}}-\frac{\sin \left(t \omega_{n}^{-}\right)}{\omega_{n}^{-}}\right), \\
& \frac{1}{\sqrt{2 \pi}}\left(\cos \left(t \omega_{n}^{-}\right)+\cos \left(t \omega_{n}^{+}\right)\right), \\
& \left.\frac{n}{\sqrt{2 \pi}} \sqrt{\frac{\beta}{\alpha}}\left(\cos \left(t \omega_{n}^{+}\right)-\cos \left(t \omega_{n}^{-}\right)\right)\right\}_{n^{2} \geq \sqrt{\alpha \beta}}
\end{aligned}
$$

is bounded and boundedly invertible in $L^{2}(0, T)$ for any $T>0$. In fact, $\mathcal{F}_{1}=\mathcal{B} \mathcal{E}_{1}$, where $\mathcal{B}$ is a diagonal block matrix of the form (17), with

$$
\begin{aligned}
B_{n} & =\frac{1}{\sqrt{2 \pi}}\left(\begin{array}{cc}
n\left(\frac{\omega_{n}^{+}+\omega_{n}^{-}}{\omega_{n}^{+} \omega_{n}^{-}}\right) & n \frac{\left(\omega_{n}^{-}-\omega_{n}^{+}\right)}{\omega_{n}^{-}} \\
\sqrt{\frac{\beta}{\alpha}} n^{2} \frac{\left(\omega_{n}^{-}-\omega_{n}^{+}\right)}{\omega_{n}^{+} \omega_{n}^{-}} & \sqrt{\frac{\beta}{\alpha}} n^{2} \frac{\left(\omega_{n}^{+}-\omega_{n}^{-}\right)}{\omega_{n}^{-}} \\
0 & 0 \\
0 & 0 \\
0 & 0 \\
0 & \omega_{n}^{-}-\omega_{n}^{+} \\
0 & \sqrt{\frac{\beta}{\alpha}} n\left(\omega_{n}^{+}-\omega_{n}^{-}\right)
\end{array}\right) .
\end{aligned}
$$

Observe that for every $n$

$$
\operatorname{det} B_{n}=\frac{n^{4}}{\pi^{2}} \frac{\beta\left(\omega_{n}^{+}-\omega_{n}^{-}\right)^{2}}{\alpha \omega_{n}^{-} \omega_{n}^{+}}>0
$$

and

$$
\lim _{n \rightarrow \infty} \operatorname{det} B_{n}=\frac{\beta^{2}}{\pi^{2}} .
$$

The analysis of the basis property of the corresponding family in this case can be accomplished according to the plan presented in Section 3.1. First, we notice that $\omega_{n}^{+}-$ $\omega_{n}^{-}=O(1 / n)$. Due to the results of Avdonin and Ivanov (2001) as well as Avdonin and Moran (2001a), the family $\mathcal{E}:=\mathcal{E}_{\sqrt{\alpha \beta}} \cup \mathcal{E}_{1}$ forms an $\mathcal{L}$-basis in $L^{2}(0, T)$ for $T \geq 4 \pi$ provided (6) never occurs for $m \neq n$. Therefore, $\mathcal{F}_{\sqrt{\alpha \beta}} \cup$ $\mathcal{F}_{1}$ also forms there an $\mathcal{L}$-basis.

3.3. Case $\boldsymbol{\alpha} \boldsymbol{\beta}<0$. Observe that in this case the equality (6) never holds, and we will prove that the system (4) is exactly controllable for any time $T \geq 4 \pi$.

Let

$$
\begin{aligned}
& \delta_{n}^{+}:=\sqrt{\frac{1}{2}\left(\sqrt{n^{4}-\alpha \beta}+n^{2}\right)}, \\
& \delta_{n}^{-}:=\sqrt{\frac{1}{2}\left(\sqrt{n^{4}-\alpha \beta}-n^{2}\right)} .
\end{aligned}
$$


The solution of (11) and (12) has the form

$$
\begin{aligned}
a_{n}(t)= & \frac{\kappa_{n}}{\sqrt{n^{4}-\alpha \beta}} \\
& \times \int_{0}^{t}\left(\delta_{n}^{-} \sinh \left(\delta_{n}^{-}(t-\tau)\right) \cos \left(\delta_{n}^{+}(t-\tau)\right)\right. \\
& \left.+\delta_{n}^{+} \cosh \left(\delta_{n}^{-}(t-\tau)\right) \sin \left(\delta_{n}^{+}(t-\tau)\right)\right) u(\tau) \mathrm{d} \tau, \\
b_{n}(t)= & \frac{\beta \kappa_{n}}{2 \sqrt{n^{4}-\alpha \beta}} \\
& \int_{0}^{t}\left(\frac{\sinh \left(\delta_{n}^{-}(t-\tau)\right) \cos \left(\delta_{n}^{+}(t-\tau)\right)}{\delta_{n}^{-}}\right. \\
& \left.-\frac{\cosh \left(\delta_{n}^{-}(t-\tau)\right) \sin \left(\delta_{n}^{+}(t-\tau)\right)}{\delta_{n}^{+}}\right) u(\tau) \mathrm{d} \tau, \\
\dot{a}_{n}(t)= & \kappa_{n} \int_{0}^{t} \cosh \left(\delta_{n}^{-}(t-\tau)\right) \cos \left(\delta_{n}^{+}(t-\tau)\right) u(\tau) \mathrm{d} \tau, \\
\dot{b}_{n}(t)= & -\sqrt{-\frac{\beta}{\alpha}} \kappa_{n} \\
& \times \int_{0}^{t} \sinh \left(\delta_{n}^{-}(t-\tau)\right) \sin \left(\delta_{n}^{+}(t-\tau)\right) u(\tau) \mathrm{d} \tau .
\end{aligned}
$$

We see that $\delta_{n}^{+} \asymp n$ and $\delta_{n}^{-}=O(1 / n)$.

Substituting $\kappa_{n}=\sqrt{2 / \pi} n$, we have

$$
\begin{aligned}
& a_{n}(t)=n \sqrt{\frac{2}{\pi\left(n^{4}-\alpha \beta\right)}} \\
& \times \int_{0}^{t}\left(\delta_{n}^{-} \sinh \left(\delta_{n}^{-}(t-\tau)\right) \cos \left(\delta_{n}^{+}(t-\tau)\right)\right. \\
& \left.+\delta_{n}^{+} \cosh \left(\delta_{n}^{-}(t-\tau)\right) \sin \left(\delta_{n}^{+}(t-\tau)\right)\right) \\
& \times u(\tau) \mathrm{d} \tau \text {, } \\
& n b_{n}(t)=\frac{\beta}{\sqrt{2 \pi}} n^{2} \int_{0}^{t}\left(\frac{\sinh \left(\delta_{n}^{-}(t-\tau)\right) \cos \left(\delta_{n}^{+}(t-\tau)\right)}{\delta_{n}^{-} \sqrt{n^{4}-\alpha \beta}}\right. \\
& \left.-\frac{\cosh \left(\delta_{n}^{-}(t-\tau)\right) \sin \left(\delta_{n}^{+}(t-\tau)\right)}{\delta_{n}^{+} \sqrt{n^{4}-\alpha \beta}}\right) u(\tau) \mathrm{d} \tau, \\
& \begin{aligned}
\dot{n}^{-1} a_{n}(t)= & \sqrt{\frac{2}{\pi}} \int_{0}^{t} \cosh \left(\delta_{n}^{-}(t-\tau)\right) \\
& \cos \left(\delta_{n}^{+}(t-\tau)\right) u(\tau) \mathrm{d} \tau,
\end{aligned} \\
& \dot{b}_{n}(t)=-n \sqrt{-\frac{\beta}{\alpha}} \sqrt{\frac{2}{\pi}} \cdot \int_{0}^{t} \sinh \left(\delta_{n}^{-}(t-\tau)\right) \\
& \times \sin \left(\delta_{n}^{+}(t-\tau)\right) u(\tau) \mathrm{d} \tau \text {. }
\end{aligned}
$$

Let us consider the family

$$
\begin{aligned}
\mathcal{E}=\left\{e^{\left(\delta_{n}^{-}+i \delta_{n}^{+}\right) t}, \frac{e^{\left(\delta_{n}^{-}+i \delta_{n}^{+}\right) t}-e^{-\left(\delta_{n}^{-}-i \delta_{n}^{+}\right) t}}{2 \delta_{n}^{-}},\right. \\
\left.e^{\left(\delta_{n}^{-}-i \delta_{n}^{+}\right) t}, \frac{e^{\left(\delta_{n}^{-}-i \delta_{n}^{+}\right) t}-e^{-\left(\delta_{n}^{-}+i \delta_{n}^{+}\right) t}}{2 \delta_{n}^{-}}\right\}_{n \in \mathbb{N}} .
\end{aligned}
$$

One can check that the operator which maps $\mathcal{E}$ to the family

$$
\begin{aligned}
\mathcal{F}_{1}= & \left\{n \sqrt { \frac { 2 } { \pi } } \left(\frac{\delta_{n}^{+} \cosh \left(\delta_{n}^{-} t\right) \sin \left(\delta_{n}^{+} t\right)}{\sqrt{n^{4}-\alpha \beta}}\right.\right. \\
& \left.+\frac{\delta_{n}^{-} \cos \left(\delta_{n}^{+} t\right) \sinh \left(\delta_{n}^{-} t\right)}{\sqrt{n^{4}-\alpha \beta}}\right), \\
& \frac{n^{2} \beta}{\sqrt{2 \pi}}\left(\frac{\left.\cosh \left(\delta_{n}^{-} t\right) \sin \left(\delta_{n}^{+} t\right)\right)}{\delta_{n}^{+} \sqrt{n^{4}-\alpha \beta}}\right. \\
& \left.-\frac{\sinh \left(\delta_{n}^{-} t\right) \cos \left(\delta_{n}^{+} t\right)}{\delta_{n}^{-} \sqrt{n^{4}-\alpha \beta}}\right), \\
& \sqrt{\frac{2}{\pi}} \cosh \left(\delta_{n}^{-} t\right) \cos \left(\delta_{n}^{+} t\right), \\
& \left.\sqrt{\frac{2}{\pi}} n \sqrt{-\frac{\beta}{\alpha}} \sinh \left(\delta_{n}^{-} t\right) \sin \left(\delta_{n}^{+} t\right)\right\}
\end{aligned}
$$

is bounded and boundedly invertible in this space. In fact, $\mathcal{F}_{1}=\mathcal{B} \mathcal{E}$, where $\mathcal{B}$ is a diagonal block matrix of the form (17) with

$$
\begin{gathered}
B_{n}=\sqrt{\frac{2}{\pi}}\left(\begin{array}{cc}
-\frac{i n \delta_{n}^{+}}{2 \sqrt{n^{4}-\alpha \beta}} & \frac{n \delta_{n}^{-}\left(\delta_{n}^{-}+i \delta_{n}^{+}\right)}{2 \sqrt{n^{4}-\alpha \beta}} \\
\frac{\sqrt{\pi}}{8} \frac{i n^{2} \beta}{\sqrt{n^{4}-\alpha \beta} \delta_{n}^{+}} & -\frac{\sqrt{\pi}}{8} \frac{i n^{2} \beta\left(\delta_{n}^{-}+i \delta_{n}^{+}\right)}{\sqrt{n^{4}-\alpha \beta} \delta_{n}^{+}} \\
\frac{1}{2} & -\frac{\delta_{n}^{-}}{2} \\
0 & \frac{1}{2} i n \sqrt{-\frac{\beta}{\alpha}} \delta_{n}^{-}
\end{array} \mid\right. \\
\operatorname{det} B_{n}=\frac{\left.\begin{array}{cc}
\frac{i n \delta_{n}^{+}}{2 \sqrt{n^{4}-\alpha \beta}} & \frac{n \delta_{n}^{-}\left(\delta_{n}^{-}-i \delta_{n}^{+}\right)}{2 \sqrt{n^{4}-\alpha \beta}} \\
-\frac{\sqrt{\pi}}{8} \frac{i n^{2} \beta}{\sqrt{n^{4}-\alpha \beta} \delta_{n}^{+}} & \frac{\sqrt{\pi} \frac{i n^{2} \beta\left(\delta_{n}^{-}-i \delta p\right)}{8}}{\sqrt{n^{4}-\alpha \beta} \delta_{n}^{+}} \\
\frac{1}{2} & -\frac{\delta_{n}^{-}}{2} \\
0 & \sqrt{2} i n \sqrt{-\frac{\beta}{\alpha}} \delta_{n}^{-}
\end{array}\right)}{2 \pi^{2} \sqrt{n^{4}-\alpha \beta} \sqrt{\sqrt{n^{4}-\alpha \beta}+n^{2}}} \neq 0
\end{gathered}
$$

and

$$
\lim _{n \rightarrow \infty} \operatorname{det} B_{n}=\frac{\beta|\beta|}{4 \pi^{2}} .
$$

Due to the results of Avdonin and Ivanov (2001) as well as Avdonin and Moran (2001a), $\mathcal{E}$ forms an $\mathcal{L}$-basis in $L^{2}(0, T)$ for $T \geq 4 \pi$ and then $\mathcal{F}$, too. 


\section{Theorem proofs}

Proof. (Theorem 1) From the results of Sections 3.1-3.3 it follows that for any $\alpha, \beta \in \mathbb{R}(\beta \neq 0)$ the family $\mathcal{F}_{N}=$ $\left\{f_{n}(t)\right\}_{n \geq N}$ forms an $\mathcal{L}$-basis in $L^{2}(0, T)$ for $T \geq 4 \pi$ and sufficiently large $N$. Then Theorem 1 follows from the results of (Avdonin and Ivanov, 1995, Theorems I.2.1a and III.3.10a). For $\beta=0$ the statement of Theorem 1 is obviously true.

Proof. (Theorem 21i)) If (6) never occurs, the family $\mathcal{F}$ forms an $\mathcal{L}$-basis in $L^{2}(0, T)$ for $T \geq 4 \pi$. The statement of Theorem 2 (i) now follows from the work of Avdonin and Ivanov (1995, Theorems 1.2.1a and III.3.10a).

Proof. (Theorem 2(ii)) If $\alpha \beta>0$ (see Section 3.2) for some $\alpha$ and $\beta$, there may exist a finite number of pairs $(m, n)$ satisfying equality (6). In this case the family $\mathcal{F}$ is clearly linearly dependent since some function (or functions) repeats twice in this family. Then, according to Avdonin and Ivanov (1995, Theorems I.2.1e and III.3.10e), our system is not approximately controllable for any $T>0$.

Proof. (Theorem 2(iii)) If $T<4 \pi$, then in all the cases considered in Section 3 the corresponding family $\mathcal{E}$ does form an $\mathcal{L}$-basis in $L^{2}(0, T)$. Moreover, it was proved by Avdonin and Moran (2001a) that there exists a proper subfamily $\mathcal{E}_{T} \subset \mathcal{E}$ constituting a Riesz basis in $L^{2}(0, T)$. Then the family $\mathcal{F}_{T}=\mathcal{B} \mathcal{E}_{T}$ is also a Riesz basis in $L^{2}(0, T)$ since $\mathcal{B}$ is a linear isomorphism in this space. Then we again can use Theorems I.2.1e and III.3.10.e of Avdonin and Ivanov (1995) to demonstrate that our system is not approximately controllable in $L^{2}(0, T)$ if $T<4 \pi$.

Theorem 3 follows evidently from Theorem 1.2 but really it was proved independently.

\section{Conclusions}

In this paper we described exactly controllable spaces (with sharp Sobolev scale exponents) for a coupled hyperbolic system, obtained a sharp estimate of controllability time, and gave explicit descriptions of (at most a finite number) non-controllable situations.

The following two conjectures may be considered our future plans.

Conjencture 1. We work on the one dimensional setting here, but we expect that similar results (exact controllability in the same order Sobolev spaces with at most a finite number of non-controllable cases) are valid also in a multi-dimensional situation.

Conjencture 2. Observe that the control time given in Theorem $2(i)$ is the doubled control time needed to control from the boundary a single (one dimensional) wave equation. So we can expect that in the general framework (in several dimensions) the control time for two coupled wave equations with a single boundary control will be the double of the geometric time required for a single wave equation controlled from the boundary.

\section{Acknowledgment}

The first author expresses his gratitude to the Institute of Physics and Mathematics of Universidad Michoacana de San Nicolás de Hidalgo, México, for its hospitality. This work was completed with the support of the Visiting Distinguished Professor 2011 grant of the Mexican Academy of Sciences and partially supported by the Conacyt grant no. 153184, PROMEP, PAPIIT-UNAM project IN108211, CIC-UMSNH, México.

\section{References}

Alabau-Boussouira, F. (2003). A two-level energy method for indirect boundary observability and controllability of weakly coupled hyperbolic systems, SIAM Journal on Control and Optimization 42(3): 871-906.

Alabau-Boussouira, F. and Leautaud, M. (2011). Indirect controllability of locally coupled systems under geometric conditions, Comptes Rendus Mathematique 349(7-8): 395-400.

Ammar-Kohdja, A., Benabdallah, M., González-Burgos, L. and de Teresa, L. (2011). Recent results on the controllability of coupled parabolic problems: A survey, Mathematical Control and Related Fields 1(3): 267-306

Avdonin, S.A. and Ivanov, S.A., (1995). Families of Exponentials. The Method of Moments in Controllability Problems for Distributed Parameter Systems, Cambridge University Press, New York, NY.

Avdonin, S.A. and Ivanov, S.A. (2001). Exponential Riesz bases of subspaces and divided differences, St Petersburg Mathematical Journal 13(3): 339-351.

Avdonin, S. and Moran, W. (2001a). Ingham-type inequalities and Riesz bases of divided differences, International Journal of Applied Mathematics and Computer Science 11(4): 803-820.

Avdonin, S. and Moran, W. (2001b). Simultaneous control problems for systems of elastic strings and beams, Systems and Control Letters 44(2): 147-155.

Avdonin, S. and Pandolfi, L. (2011). Temperature and heat flux dependence independence for heat equations with memory, in R. Sipahi, T. Vyhlidal, S.-I. Niculescu and P. Pepe (Eds.), Time Delay Systems-Methods, Applications and New Trends, Lecture Notes in Control and Information Sciences, Vol. 423, Springer-Verlag, Berlin/Heidelberg, pp. 87-101.

Biot, M. (1962). Generalized theory of acoustic propagation in porous dissipative media, The Journal of the Acoustical Society of America 34(9): 1254-1264. 
Bodart, O. and Fabre, C. (1995). Controls insensitizing the norm of the solution of a semilinear heat equation, Journal of Mathematical Analysis and Applications 195(3): 658-683.

Dáger, R. (2006). Insensitizing controls for the 1-D wave equation, SIAM Journal on Control and Optimization 45(5): 1758-1768.

El Jai, A. and Hamzaoui, H. (2009). Regional observation and sensors, International Journal of Applied Mathematics and Computer Science 19(1): 5-14, DOI: 10.2478/v10006-009-0001-y.

Hansen, S. and Zuazua E. (1995). Exact controllability and stabilization of a vibrating string with an interior point mass, SIAM Journal on Control and Optimization 33(5): 1357-1391.

Holger, S., Frehner, M. and Schmalholz S. (2010). Waves in residual-saturated porous media, in G.A. Maugin and A.V. Metrikine (Eds.), Mechanics of Generalized Continua, Advances in Mechanics and Mathematics, Vol. 21, Springer, New York, NY, pp. 179-187.

Kavian, O. and de Teresa, L. (2010). Unique continuation principle for systems of parabolic equations, ESAIM: Control, Optimisation and Calculus of Variations 16(2): 247-274.

Khapalov, A. (2010). Source localization and sensor placement in environmental monitoring, International Journal of Applied Mathematics and Computer Science 20(3): 445-458, DOI: 10.2478/v10006-010-0033-3.

Leonard, E. (1996). The matrix exponential, SIAM Review 38(3): 507-512.

Lions, J.L. (1989). Remarques préliminaires sur le contrôle des systèmes à données incomplètes, XI Congreso de Ecuaciones Diferenciales y Aplicaciones (CEDYA), Málaga, Spain pp. 43-54.

Najafi, M. (2001). Study of exponential stability of coupled wave systems via distributed stabilizer, International Journal of Mathematics and Mathematical Sciences 28(8): 479-491.

Najafi, M., Sarhangi G.R. and Wang H. (1997). Stabilizability of coupled wave equations in parallel under various boundary conditions, IEEE Transactions on Automatic Control 42(9): 1308-1312.

Pandolfi, L. (2009). Riesz system and the controllability of heat equations with memory Integral Equations and Operator Theory 64(3): 429-453.

Rosier, L. and de Teresa, L. (2011). Exact controllability of a cascade system of conservative equations, Comptes Rendus Mathematique 349(5): 291-296.
Russell, D. (1978). Controllability and stabilizability theory for linear partial differential equations, SIAM Review 20(4): 639-739.

Tebou, L. (2008). Locally distributed desensitizing controls for the wave equation, Comptes Rendus Mathematique 346(7): 407-412.

de Teresa, L. (2000). Insensitizing controls for a semilinear heat equation, Communications in Partial Differential Equations 25(1-2): 39-72.

Uciński, D. and Patan, M. (2010). Sensor network design for the estimation of spatially distributed processes, International Journal of Mathematics and Mathematical Sciences 20(3): 459-481, DOI: 10.2478/v10006-010-0034-2.

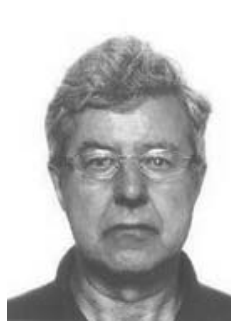

Sergei Avdonin received his Ph.D. in mathematics in 1977 from St. Petersburg State University, Russia, and then worked at this university for more than twenty years. He has also worked at the Flinders University of South Australia and the University of Tennessee Chattanooga. Currently, he is a professor of mathematics at the University of Alaska Fairbanks. His research interests include nonharmonic Fourier series, control theory of partial differential equations and inverse problems of mathematical physics.

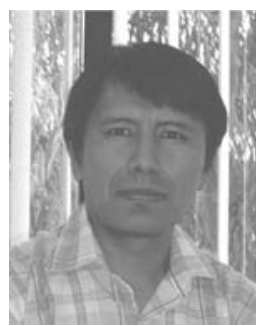

Abdon Choque Rivero was born in Bolivia in 1965. He received the M.Sc. degree in mathematics from Kharkov State University, former Soviet Union, and the Ph.D. in mathematics from Leipzig University, Germany, in 1992 and 2002, respectively. Currently, he works at Universidad Michoacana de San Nicolás de Hidalgo, Morelia, Michocán, México. He has done research in control theory and matrix moment problem.

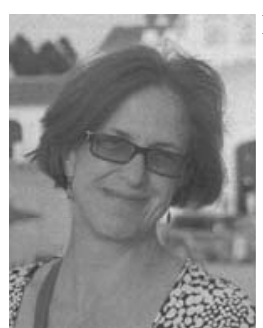

Luz de Teresa was born in Mexico City in 1965 She studied mathematics at UNAM (Universidad Nacional Autónoma de México). She obtained her Ph.D. in 1995 in applied mathematics at Universidad Complutense de Madrid, Spain. Since 1995 she has been working at the Institute of Mathematics, UNAM. Her main contributions are on the controllability of coupled parabolic equations.

Received: 18 September 2012 Revised: 25 March 2013 